\title{
AFM and Van der Pauw Measurements of Carbon Nanoparticles Prepared from Kerosene
}

\author{
Tanisha Mehreen ${ }^{1}$, Jahirul Islam ${ }^{1}$, Mohammad Abul Hossain ${ }^{2}$, \\ Khandker Saadat Hossain ${ }^{1}$
}

\author{
${ }^{1}$ Department of Physics, University of Dhaka, Dhaka-1000, Bangladesh \\ ${ }^{2}$ Department of Chemistry, University of Dhaka, Dhaka-1000, Bangladesh
}

\begin{abstract}
In the present study, the structural and electrical properties of carbon nanoparticles, prepared from kerosene has been investigated with the help of Thermogravimetric Analysis (TGA), Atomic Force Microscope (AFM) and Ecopia Hall-effect measurement system. The spherical carbon particles size was observed with the help of Atomic Force Microscope (AFM) and the size found $80 \mathrm{~nm}$ in diameter as an aggregated particles. The electrical parameters such as resistivity, conductivity, magneto-resistance, mobility, and average Hall coefficient were measured by Ecopia Hall effect measurement system by four-point probe method at a suitable condition. The IVcharacteristic curve of Carbon Nanoparticle Thin Film (CNTF) was observed by a source-meter and a current rectifying behavior at the $\mathrm{Ag} / \mathrm{CNTF}$ interface was found. The CNPs synthesized from kerosene was found as an $n$ type semiconductor and other electrical properties were compared with CNPs prepared from diesel in the previous study.
\end{abstract}

Keywords: Carbon Nanoparticles,Atomic Force Microscope, Van Der Pauw, Rectifier, Thin Film

\section{Introduction}

Nano-structured materials can be made with unique nanostructures and properties. These materials may be metals, semiconductors, metal oxides, organic materials or biomaterials. Because of the recent discovery of several new nanoscale graphitic materials with very unique properties, nanomaterials of carbon, especially graphitic nanomaterials, have sparked enormous interest in the last decade (Iijima, 1991; Sano et al., 2001; Novoselov et al., 2004).

The properties of solid materials change significantly when their dimensions are reduced to nanometer scale. For semiconductors, this transition occurs when the particle sizes are comparable to the de Broglie wavelength of the electron, to the mean free path of the exciton, or to the wavelength of the phonons. Nanoparticles i.e. particles in the nanometer range are size quantized. As the particle size gets smaller, larger portion of their constituent atoms are located at the surface. But in the bulk materials fewer atoms are at or near the surface. Nanomaterials exhibit quite different surface properties such as energy levels, electronic structure and reactivity from the bulk material properties (Mitin et al., 2010). Due to the nanometer size, many of the mechanical, electrical, thermal, optical, chemical and other properties are modified, enabling unique applications.
The nanoparticles usually have a diameter less than $100 \mathrm{~nm}$. Carbon nanoparticles are nano-sized carbon elements created through various methods. Depending on the manufacturing techniques used to create the particles, the physical properties of carbon nanoparticles change. Various synthesis methods such as nitric acid oxidation of carbon soot which afforded a light emitting property of carbon particles of size 2-6 nm (Ray et al., 2009), a green synthesis of carbon nanoparticles from commercial grade honey with photo-acoustic imaging application (Wu, 2013), synthesis of carbon nanoparticles by laser irradiation (Hu et al., 2009) have been used. An inexpensive and affordable synthesis method is much more desired than high-cost synthesis methods.

A cost-effective method of synthesis from an affordable material such as kerosene has been successfully done recently. Nano-structured nonuniform spherical carbon was synthesized from incomplete combustion of kerosene with size between 20-100 nm observed by Scanning Electron Microscope (SEM).The elemental analysis of prepared material was done with EDX, XRD and FTIR analysis (Hossain et al., 2013). The present study focuses on further analysis of carbon nanoparticles synthesized from kerosene, in order to find potential application of 
carbon nanoparticles synthesized from low-cost method and inexpensive materials such as kerosene.

Moreover, a similar cost-effective method was used to synthesize carbon nanoparticles. Using Diesel, which is an affordable and cheap material, carbon nanoparticles of size 10 to $80 \mathrm{~nm}$ in diameter was synthesized. The electrical property study revealed a rectifying behavior which confirms a potential application for the CNPs synthesized from low-cost method (Hossain et al., 2016). In the current study the electrical property measurements will be compared with that of CNPs synthesized from diesel in order to determine which will be better suited for electrical applications.

In the present study, structural and electrical properties study of Carbon nanoparticles synthesized from kerosene were done with Thermogravimetric analysis (TGA), Atomic Force Microscope (AFM) and Van der Pauw Hall effect measurement system. Thermogravimetric analysis (TGA) was done to investigate the existence of functional group and thermal stability of the synthesized carbon nanoparticles.Atomic Force Microscope (AFM) studies on surface morphology, size distribution, roughness of the surface, and height of the surfacewas inspected scrupulously. Van der Pauw Hall-effect measurement system for the measurement of electrical properties such as conductivity, Hall-effect measurement etc. and source meter unit for the $I-V$ characteristics of carbon nanoparticles was carried out searching for its practical application. Moreover, a comparison between the results of electrical property studies of both CNPs synthesized from Kerosene and Diesel were done.

\section{Experimental Section \\ 2.1 Materials}

\subsubsection{Sample preparation}

Kerosene, a commonly used fuel in Bangladesh, was collected from local market in Dhaka city. Thoroughly cleaned and dried glass round bottom flax, lamp and lamp wick was used. Using Kerosene as a fuel, the lamp was fired and the round glass bottom flax was placed over it in order to prevent the excess air oxygen. As the kerosene burned in the lamp, a black colored material was prepared and was deposited inside of the glass round bottom flax. Then, the deposited materials were collected in a dry bottle and stored in a desiccator (Hossain et al., 2013).

\subsubsection{Thin film preparation}

Thin films were prepared in two different ways- spin coating and drop casting method. In order to acquire AFM image of CNP, the samples were prepared by spin coating on the $\mathrm{SiO}_{2}$ insulating substrates. A CNP solution $\left(10^{-5} \mathrm{~mol} / \mathrm{L}\right)$ in DMSO was deposited on $\mathrm{SiO}_{2}$ substrate and spin coated (5000 rpm for 30 seconds).
The sample was dried and mounted on the microscope chamber. The surface morphology was extensively studied by AFM (Nanosurf, FlexAFM, Switzerland). For the electrical property measurements, the CNP solution was drop casted on the chemically cleaned $\mathrm{SiO}_{2}$ substrate and was kept in the oven at a temperature of $100^{\circ} \mathrm{C}$ for $30-40$ minutes. The casting of solution was controlled carefully concerning that over dropping might result thicker films. The obtained thin film was chemically and thermally stable, homogeneous and well-adhered to the substrate as well.

\subsection{Methods}

\subsubsection{Thermal gravimetric analysis}

Thermal Gravimetric Analysis (TGA) of the prepared carbon nanoparticles for thermal stability was performed using a TA instrument TGA-50H.tad under a Nitrogen atmosphere. The heating rate was $10^{\circ} \mathrm{C} /$ min and the flow rate for nitrogen environment was 10 $\mathrm{mL} / \mathrm{min}$. All the experiments were conducted in the temperature range from 23 to $800^{\circ} \mathrm{C}$.

\subsubsection{Surface morphology study with AFM}

In order to study the surface morphology of CNP, certain amounts of CNP suspension in DMSO was firstly drop casted onto chemically and ultrasonically cleaned $\mathrm{SiO}_{2}$ substrates and spin coated $(5000 \mathrm{rpm}$ for 30 seconds). To remove the thermally unstable molecular conformations, the sample was heated at $100^{\circ} \mathrm{C}$ for 5 to 10 minutes. After that, the samples were loaded into the microscope. Atomic force microscopy (AFM) measurements were performed using non-contact mode. The concentrations of the solutions were $10^{-5}$ to $10^{-9} \mathrm{~mol} / \mathrm{L}$. The topography measurements resolution was at $256 \times 256$ points.

\subsubsection{Electrical properties measurement}

The resistivity, conductivity, magneto resistance, mobility and average Hall co-efficient measurements were conducted in lateral direction of the carbon nanoparticle thin film (CNTF) by 'Ecopia Hall-effect measurement system' by 4-point Van der Pauw approach at ambient condition.

For current-voltage $(I-V)$ measurements, wiring was made by soldering two silver (Ag) electrodes each of 1 $\mathrm{mm}$ wide at the two edges of the film surface. Electrical measurements were carried out at by using 'Keithley' 2401 Source Meter for current and voltage measurements at ambient condition. LabTracer software was used for the current-voltage measurements.

\section{Results and Discussion}

\subsection{TGA Analysis}

In order to investigate the existence of functional group and thermal stability of the synthesized carbon nanoparticles, we performed Thermogravimetric 
Analysis (TGA) experiment. Results of TGA data are illustrated in Figure 1. The Figure shows that all composites and pure carbon nanoparticles begin to degrade at $238^{\circ} \mathrm{C}$ with $2.37 \%$ weight loss. First weight loss for carbon nanoparticles occurred between $238^{\circ} \mathrm{C}$ and $400^{\circ} \mathrm{C}$ and a rapid weight loss was observed around $508^{\circ} \mathrm{C}$. However, the first weight loss peak was observed in the range of $500^{\circ} \mathrm{C}$ to $550^{\circ} \mathrm{C}$. The temperature of maximum rate of oxidation is $695^{\circ} \mathrm{C}$ for the nanoparticle sample, $645^{\circ} \mathrm{C}$ for graphite and $420^{\circ} \mathrm{C}$ for $\mathrm{C}_{60}$ (Pang et al., 1993). In Figure 1, we found that the maximum rate of oxidation was $633^{\circ} \mathrm{C}$ for carbon nanoparticles, which were close to graphite. The slight deviation from the expected value indicated that a small amount of amorphous carbon was present in this sample. However, the prepared carbon nanoparticles are about $72 \%$ pure, which was proved in the EDX analysis of the prepared carbon nanoparticles sample (Hossain et al., 2013).

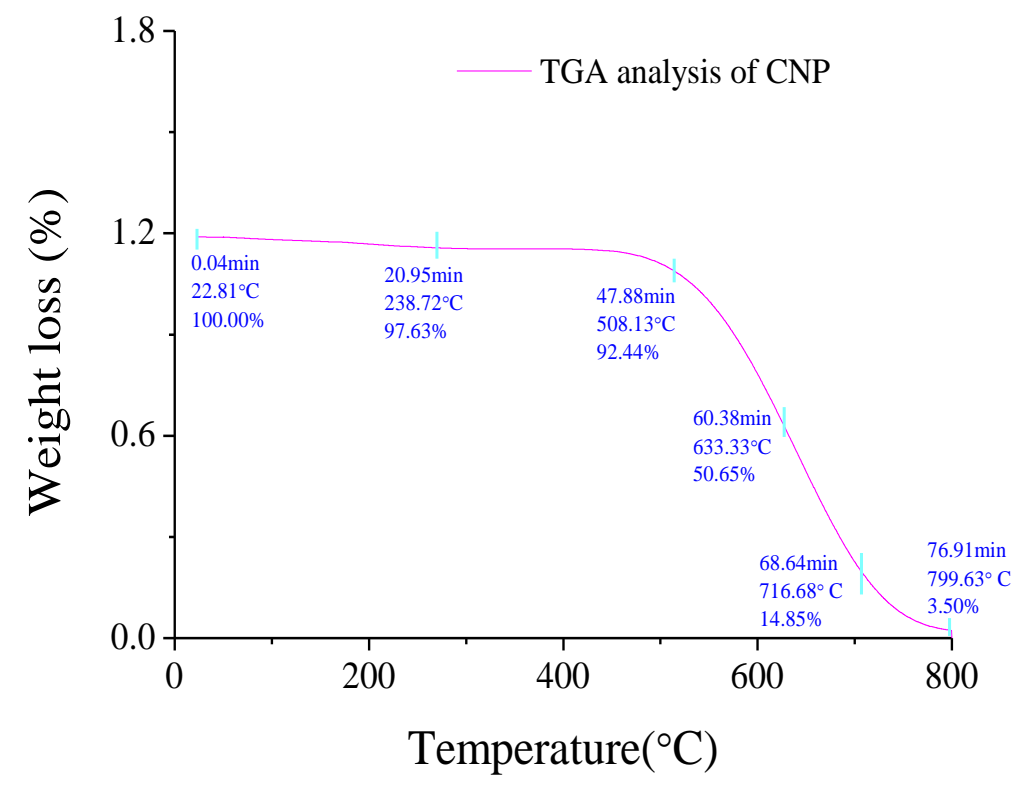

Figure 1: Thermogravimetric analysis of carbon nanoparticles prepared from kerosene.

\subsection{AFM Measurement}

The surface morphology was extensively studied by AFM. Figure 2(a) shows a standard AFM topography image of CNP solution of concentration $10^{-5} \mathrm{~mol} / \mathrm{L}$ deposited $\mathrm{SiO}_{2}$ substrate, where the shape of particles are mostly spherical in nature. Figure 2(b) indicates the 3D view of Figure 2(a), where the particles are aggregated with each other. In order to remove the aggregation of the particles and to observe the size and roughness of an isolated particle, the solution was diluted to $10^{-7} \mathrm{~mol} / \mathrm{L} \mathrm{CNP}$.

Figure 3(a) represents a standard AFM topography image of CNP for $10^{-7} \mathrm{~mol} / \mathrm{L}$ at $10.3 \mu \mathrm{m}$ scale measurement, where the particles are still aggregated with each other but the shape of the particle is also observed to be mostlyspherical. Figure 3(b) shows the 3D image of Figure 3(a). In order to observe a single isolated particle, the concentration was diluted even further.

Figure 4 shows the AFM topographical image of a single CNP for $10^{-9} \mathrm{~mol} / \mathrm{L} \mathrm{CNP}$ solution. At higher concentrations like $10^{-5} \mathrm{~mol} / \mathrm{L}$ and $10^{-7} \mathrm{~mol} / \mathrm{L}$ solutions, it was very difficult to observe the AFM topographical image of single CNP particle at nano-scale due to the aggregation of the particles in the prepared CNP solution. However, at the concentration of $10^{-9} \mathrm{~mol} / \mathrm{L}$ CNP solution, it was possible to investigate the size of the isolated CNP particle with the help of AFM and found to be $80 \mathrm{~nm}$. In the Figure, 4(a) shows the 2D AFM topographical image of a single particle; Figure 4(b) is the 3D view of Figure 4(a) and Figure 4(c) is the 2D line profile recorded along the solid line in Figure 4(a). The 2D profile in Figure 4(c) indicates that the size (height) of the particle is about $\sim 4.5 \mathrm{~nm}$. 


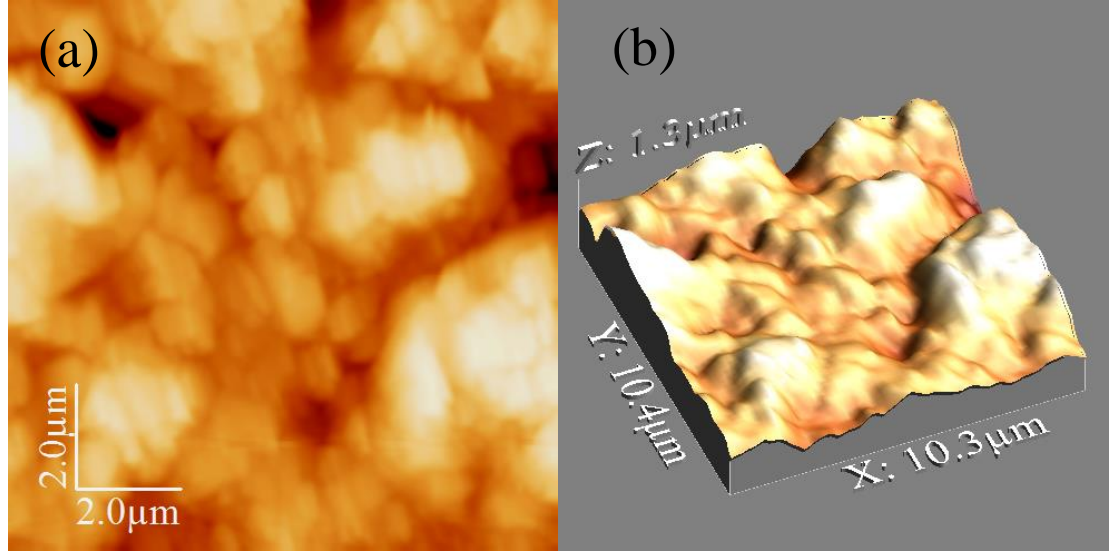

Figure 2: AFM Topographies on $\mathrm{SiO}_{2}$ surface for $10^{-5} \mathrm{~mol} / \mathrm{L}$ CNP solution: (a) Distribution of CNP deposited on $\mathrm{SiO} \mathrm{O}_{2}$ substrate (b) A 3D image of figure (a)
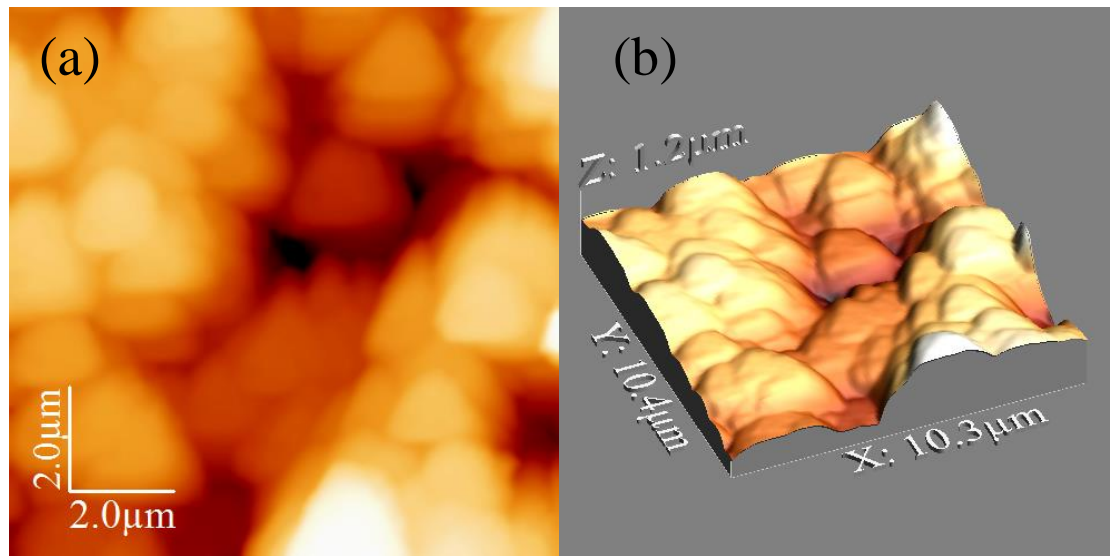

Figure 3: AFM topographies on $\mathrm{SiO}_{2}$ surface for $10^{-7} \mathrm{~mol} / \mathrm{L} \mathrm{CNP}$ solution: (a) distribution of CNP deposited on $\mathrm{SiO}_{2}$ substrate (b) A 3D image of figure (a)
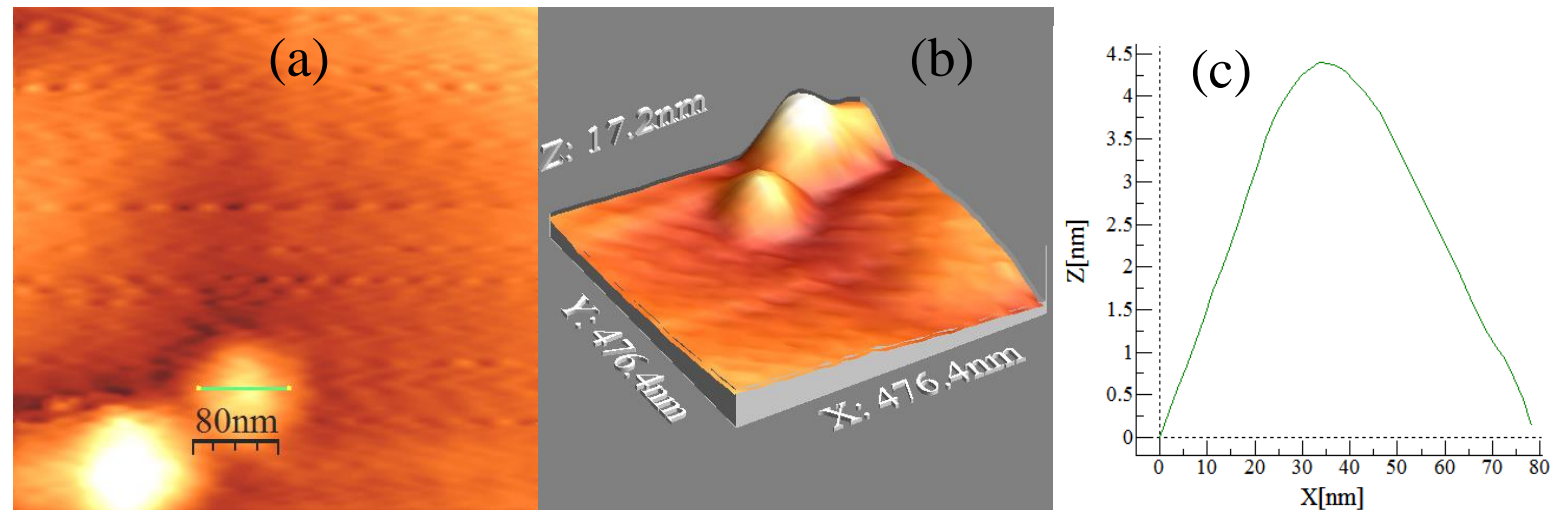

Figure 4: A high-resolution AFM image of a single isolated $\mathrm{CNP}$ on $\mathrm{Si} \mathrm{O}_{2}$ surface. (a) A 2D Topographical image (b) A 3D view of figure (a), (c) A 2D line profile recorded along the dashed line in figure 4(a) as a specified particle.

\subsection{Electrical Properties Measurement}

For electrical property measurement of Carbon Nanoparticles (CNP) thin film, the film was prepared by drop casting method. The prepared CNP solution of $10^{-3} \mathrm{~mol} / \mathrm{L}$ was drop cast onto the chemically and ultrasonically cleaned $\mathrm{SiO}_{2}$ substrate and after that keeping it in the oven at $100^{\circ} \mathrm{C}$ for $30-40$ minutes. Electrical measurements were done with Hall-effect measurement system and "four-point measurement system" at the suitable condition. The results of electrical properties of the carbon nanoparticles thin film under the two measurement systems is given in Table- 1 .

It is found that the resistivity of this carbon nanoparticles thin film is higher than that of graphitic carbon by at least three orders of magnitudes; whereas the conductivity is at least three orders of magnitudes 
lower than that of graphitic carbon at the basal plane. It is also observed that the hole mobility of this carbon nanoparticles thin film is two orders of magnitude and the bulkconcentration is five orders of magnitude less than that of graphitic carbon.

The $I-V$ characteristic curve of the carbon nanoparticle thin film is measured with the source meter unit (SMU). Figure 5 shows the DC nonlinear $I-V$ characteristic curve of $\mathrm{Ag} / \mathrm{CNTF}$ at room temperature, which was repeated five times. The curve is obtained from the Silver $(\mathrm{Ag})$ electrode and CNTF interface, where the voltage was varied from $-10 \mathrm{~V}$ to $+10 \mathrm{~V}$, at room temperature. The measurements were repeated five times and presented on the same graph.

When a metal (Ag) contact with an n-type semiconductor then a metal-semiconductor (M-S) junction is created. It shows rectifying or nonrectifying behavior. In our study, we found a smaller amount of current in the reverse bias while a large amount of current is found in the forward bias. This result represents the rectifying behavior of Ag/CNTF interface. This decrease in current in the reverse bias is due to the increase of depletion width in the metalsemiconductor (M-S) junction. The origin of this rectifying behavior on the $\mathrm{Ag} / \mathrm{CNTF}$ interface indicates a Schottky diode. From the Hall Effect data, CNTF is found to be an n-type semiconductor. According to $n$-type Schottky diode contact depends on the work function of the metal and semiconductor. As the work function of the metal is greater than the work function of semiconductor, the electrons in metal have lower potential energy than the electrons in the semiconductor. As a result, in the reverse bias, the Schottky barrier height increases with decreasing the work function of the metal electrode, which is responsible for decreasing of current than the forward biascurrent.

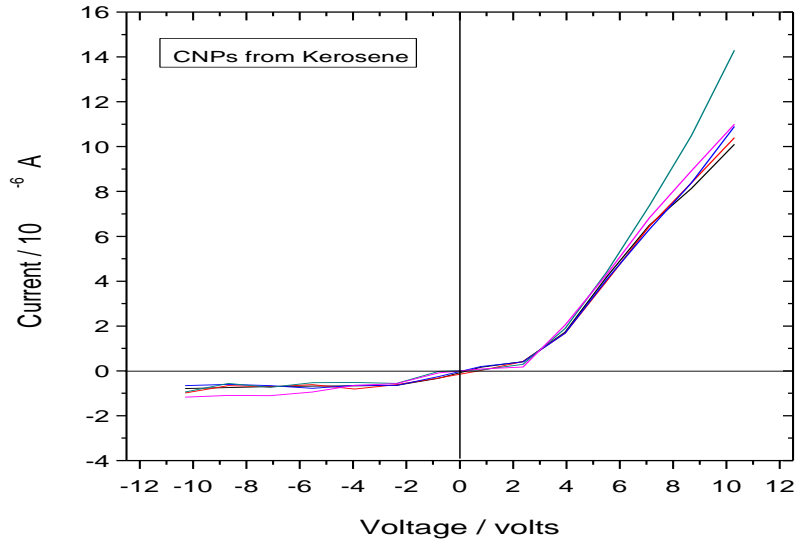

Figure 5: $I-V$ Characteristic curve of Ag/CNTF Layered Structure (from kerosene) showing current rectifying behavior.

In Table-1, different parameters of electrical properties for CNPs from kerosene and diesel(Hossain et al., 2016) are compared. It is found that the thin film of CNPs from diesel has lower resistivity than graphitic carbon (at least three orders of magnitude) (Hossain et al., 2016), whereas the thin film of CNPs from kerosene shows resistivity at least three orders of magnitude higher than graphitic carbon. As for conductivity, carbon nanoparticles thin film from diesel (Hossain et al., 2016) shows conductivity higher than graphitic carbon at basal plane by three orders of magnitude, whereas carbon nanoparticles from kerosene shows three orders of magnitude lower than graphitic carbon at basal plane. Also hole mobility and bulk concentration are lower in the case of CNPs prepared from kerosene than for CNPs from diesel. From this comparison, we can conclude that, Carbon nanoparticles prepared from kerosene is less conducting than carbon nanoparticles prepared from diesel, which might have some application as a resistor.

Table-1: Comparison between the electrical properties of CNPs prepared from kerosene and diesel.

\begin{tabular}{|c|c|c|c|c|}
\hline No. & Parameters & $\begin{array}{l}\text { Measured value for } \\
\text { CNPs from Kerosene }\end{array}$ & \multicolumn{2}{|c|}{$\begin{array}{c}\text { Measured value for } \\
\text { CNPs from Diesel (Hossain et al., 2016) }\end{array}$} \\
\hline 1. & Bulk concentration & $-5.816 \times 10^{12}$ & $9.03 \times 10^{21}$ & $\mathrm{~cm}^{-3}$ \\
\hline 2. & Sheet concentration & $-1.454 \times 10^{9}$ & $4.52 \times 10^{16}$ & $\mathrm{~cm}^{-2}$ \\
\hline 3. & Resistivity & $3.284 \times 10^{-3}$ & $4.29 \times 10^{-7}$ & $\mathrm{Ohm}-\mathrm{cm}$ \\
\hline 4. & Conductivity & $(O h m-c m)^{-1}$ & $2.33 \times 10^{6}$ & $(\mathrm{Ohm}-\mathrm{cm})^{-1}$ \\
\hline 5. & Magneto resistance & $1.256 \times 10^{7}$ & $3.64 \times 10^{-2}$ & Ohm \\
\hline 6. & Hole mobility & $3.296 \times 10^{2}$ & $1.61 \times 10^{3}$ & $c m^{2} V^{-1} s^{-1}$ \\
\hline 7. & Average Hall co-efficient & $-1.073 \times 10^{6}$ & $6.91 \times 10^{-4}$ & $\mathrm{~cm}^{3} \mathrm{C}^{-1}$ \\
\hline
\end{tabular}

Figure 6 shows $I-V$ characteristic curves at Ag/ carbon nanoparticles thin film interface for both CNPs prepared from kerosene and diesel respectively. Both curves represent rectifying behavior at $\mathrm{Ag} / \mathrm{CNTF}$ interface. From the $I-V$ characteristic curve from kerosene (red line), we can see that for positive bias, current is much higher than for negative bias. And from the $I-V$ characteristic curve from diesel (black line), it can be seen that for positive bias current is much lower than for negative bias. In conclusion, both materials can be used for rectifying purpose. 


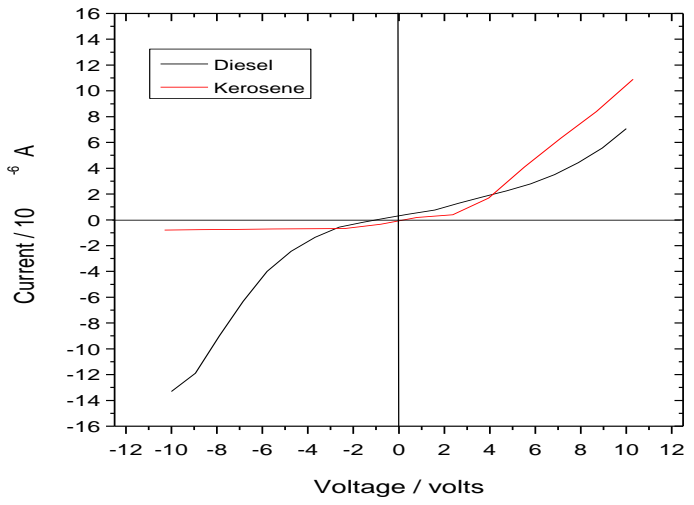

Figure6: $I-V$ characteristics of $\mathrm{Ag} / \mathrm{CNTF}$ layered structure (from diesel and kerosene) showing current rectifying behavior

\section{Conclusion}

In this study, the structural and electrical properties of carbon nanoparticles synthesized from kerosene have been investigated with TGA, AFM and Vander Pauw Hall effect measurement system. Thermogravimetric analysis (TGA) has shown that synthesized Carbon Nanoparticles are more thermally stable than Diamond. Nearly spherical shape of the particle was observed from AFM studies. A single particle of size $80 \mathrm{~nm}$ was successfully isolated in the AFM measurement. This result is similar with the SEM observation (Hossain et al., 2013). Van der Pauw Hall effect measurement system and source meter were used to investigate the electrical property of carbon nanoparticles. From this study, CNPs were found to be an $n$-type semiconductor and the conductivity is observed to be less than graphite. $I-V$ characteristic curve at the $\mathrm{Ag} / \mathrm{CNTF}$ interface were investigated and a rectifying behavior was observed. Carbon nanoparticles synthesized from kerosene have lower conductivity and higher resistance as compared with CNPs from diesel in previous study. CNPs from kerosene were found to be an $n$-type semiconductor and CNPs from diesel to be a $p$-type semiconductor. But, in both cases, at the Ag/CNTF interface, similar current rectifying behavior was observed.

\section{Acknowledgement}

The authors would like to thank ISP (International Science Program), Sweden for financially supporting this work.

\section{References}

1. Hossain, M. A., Islam, S. (2013). Synthesis of Carbon Nanoparticles from Kerosene and their Characterization by SEM/EDX, XRD and FTIR.American Journal of Nanoscience and Nanotechnology.1(2), 52-56. DOI: 10.11648/j.nano.20130102.12 https://doi.org/10.11648/j.nano.20130102.12

2. Hossain, M. A., Islam, S., Chowdhury, F. A., Mohiuddin, T. G., Uchida,K., Tamura, T., Sugawa, K., Mochida, T., Otsuki, J., Alam, M. S.(2016). Structural, mechanical, and electrical properties of carbon nanoparticles synthesized from diesel. Fullerenes, Nanotubes and Carbon Nanostructures.24(1), 43 51. DOI: 10.1080/1536383X.2015.1092436 https://doi.org/10.1080/1536383x.2015.1092436

3. Hu, S. L., Niu, K. Y., Sun, J., Yang, J., Zhao, N. Q., and Du, X. W. (2009). One-step synthesis of fluorescent carbon nanoparticles bylaser irradiation.J. Mater. Chem. 19(4), 484488. DOI: $\quad 10.1039 / \mathrm{B} 812943 \mathrm{~F}$ https://doi.org/10.1039/b812943f

4. Iijima, S. (1991).Helical microtubules of graphitic carbon.Nature.354, 56-58. https://doi.org/10.1038/354056a0

5. Mitin, V. V., Sementsov, D. I. and Vagidov, N. Z. (2010).Quantum Mechanics for nanostructures.Cambridge University Press. https://doi.org/10.1017/cbo9780511845161

6. Novoselov, K. S., Geim, A. K., Morozov, S. V., Jiang, D., Zhang, Y., Dubonos, S. V., Grigirieva, I. V. (2004). Electric field effect in atomically thin carbon films.Science.306(5696), 666-669. DOI: $\quad 10.1126 /$ science.1102896 https://doi.org/10.1126/science.1102896

7. Pang, L. S. K., Saxby, J. D., S. P. Chatfield.(1993). Thermogravimetric analysis of carbon nanotubes and nanoparticles.J.Phys. chem. 97(27), 6941-6942. DOI: 10.1021/j100129a001 https://doi.org/10.1021/j100129a001

8. Ray, S. C., Saha, A., Jana, N. R., and Sarkar, R. (2009). Fluorescent carbon nanoparticles: synthesis, characterization, and bioimaging application. J. Phys. Chem. C.113(43), 1854618551. DOI: $\quad 10.1021 / j p 905912 \mathrm{n}$ https://doi.org/10.1021/jp905912n

9. Sano, N., Wang, H., Chhowalla, M., Alexandrou, I., Amaratunga, G. A. J. (2001).Synthesis of Carbon 'Onions' in Water.Nature.414, 506-507. https://doi.org/10.1038/35107141

10. Wu, L., Cai, X., Nelson, K.., Xing, W., Xia, J., Zhang, R., Stacy, A. J., Luderer, M., Lanza, G. M., Wang, L. V., Shen, B., Pan, D. (2013). A Green Synthesis of Carbon Nanoparticle from Honey for Real-Time Photoacoustic Imaging.Nano Res. 6(5), 312-325. DOI: $\quad 10.1007 / \mathrm{s} 12274-013-0308-8$ https://doi.org/10.1007/s12274-013-0308-8 